\title{
Retinal Pigment Epithelium Transplantation in a Non- human Primate Model for Degenerative Retinal Diseases
}

\author{
Ivan Seah ${ }^{*}, 1,2$, Zengping Liu ${ }^{*}{ }^{1,3,4}$, Daniel Soo Lin Wong ${ }^{1}$, Wendy Wong ${ }^{2}$, Graham E. Holder ${ }^{1,2,5}$, Veluchamy Amutha \\ Barathi $^{1,4,6}$, Gopal Lingam ${ }^{1,2,4}$, Xinyi Su ${ }^{1,2,3,4}$, Boris V. Stanzel ${ }^{1,7,8}$ \\ ${ }^{1}$ Department of Ophthalmology, Yong Loo Lin School of Medicine, National University of Singapore ${ }^{2}$ Department of Ophthalmology, National University \\ Hospital, Singapore ${ }^{3}$ Institute of Molecular and Cell Biology (IMCB), Agency for Science, Technology and Research (A*STAR) ${ }^{4}$ Singapore Eye Research \\ Institute (SERI) ${ }^{5}$ UCL Institute of Ophthalmology ${ }^{6}$ Academic Clinical Program in Ophthalmology, Duke-NUS Medical School ${ }^{7}$ Macula Center Saar, Eye \\ Clinic Sulzbach, Knappschaft Hospital Saar ${ }^{8}$ Department of Ophthalmology, University of Bonn \\ *These authors contributed equally
}

\section{Corresponding Authors}

Xinyi Su

xinyi_su@nuhs.edu.sg

Boris V. Stanzel

boris.stanzel@kksaar.de

\section{Citation}

Seah, I., Liu, Z., Soo Lin Wong, D., Wong, W., Holder, G.E., Amutha Barathi, V., Lingam, G., Su, X., Stanzel, B.V. Retinal Pigment Epithelium Transplantation in a Non-human Primate Model for Degenerative Retinal Diseases. J. Vis. Exp. (172), e62638, doi:10.3791/62638 (2021).

\section{Date Published}

June 14, 2021

\section{DOI}

$10.3791 / 62638$

URL

jove.com/video/62638

\section{Abstract}

Retinal pigment epithelial (RPE) transplantation holds great promise for the treatment of inherited and acquired retinal degenerative diseases. These conditions include retinitis pigmentosa (RP) and advanced forms of age-related macular degeneration (AMD), such as geographic atrophy (GA). Together, these disorders represent a significant proportion of currently untreatable blindness globally. These unmet medical needs have generated heightened academic interest in developing methods of RPE replacement. Among the animal models commonly utilized for preclinical testing of therapeutics, the non-human primate (NHP) is the only animal model that has a macula. As it shares this anatomical similarity with the human eye, the NHP eye is an important and appropriate preclinical animal model for the development of advanced therapy medicinal products (ATMPs) such as RPE cell therapy.

This manuscript describes a method for the submacular transplantation of an RPE monolayer, cultured on a polyethylene terephthalate (PET) cell carrier, underneath the macula onto a surgically created RPE wound in immunosuppressed NHPs. The fovea-the central avascular portion of the macula-is the site of the greatest mechanical weakness during the transplantation. Foveal trauma will occur if the initial subretinal fluid injection generates an excessive force on the retina. Hence, slow injection under perfluorocarbon liquid (PFCL) vitreous tamponade is recommended with a dual-bore subretinal injection cannula at low intraocular pressure (IOP) settings to create a retinal bleb.

Pretreatment with an intravitreal plasminogen injection to release parafoveal RPEphotoreceptor adhesions is also advised. These combined strategies can reduce the 
likelihood of foveal tears when compared to conventional techniques. The NHP is a key animal model in the preclinical phase of RPE cell therapy development. This protocol addresses the technical challenges associated with the delivery of RPE cellular therapy in the NHP eye.

\section{Introduction}

RPE transplantation holds great promise for the treatment of inherited and acquired retinal degenerative diseases. These conditions include retinitis pigmentosa (RP, rodcone dystrophy) and advanced forms of AMD such as GA. Collectively, these disorders represent a significant proportion of currently untreatable blindness globally ${ }^{1,2}$. The advanced stages of AMD are categorized into neovascular AMD (nAMD) and GA. While there are effective treatment options for nAMD, such as anti-vascular endothelial growth factor (anti-VEGF) injections, patients with GA have limited treatment options. RP is a highly heterogeneous group of inherited retinal disorders characterized by progressive retinal photoreceptor degeneration. In some patients, the causative genetic defect is located within the RPE rather than the photoreceptors; hence, RPE replacement therapy may be an alternative strategy if gene therapy is not feasible.

There is significant interest in developing effective treatments for these conditions. In particular, RPE transplantation has been gaining traction as a potential therapeutic approach $^{3,4,5,6,7,8}$. Since the first reports on RPE transplantation appeared in the $1980 \mathrm{~s}^{9}$, the field has expanded to include various RPE cell sources, delivery strategies, and experimental models of disease and transplantation $10,11,12,13,14$. Among the various animal models, only the NHP has a 'macula lutea' with a 'fovea centralis,' an anatomic specialization at the posterior pole of the retina shared with humans. The fovea contains a very high density of cone photoreceptors enabling high-resolution central vision ${ }^{15}$. The NHP also has a similar genomic and proteomic make-up ${ }^{16}$ when compared to humans. These similarities make it an important and appropriate animal model for the study of ocular diseases that affect the human retina $^{17,18}$.

This manuscript describes a method for the submacular transplantation of an RPE xenograft, supported by a PET cell carrier, in immunosuppressed NHPs. A transvitreal technique for subretinal RPE transplantation in rabbits has been described in a previous manuscript ${ }^{19}$. However, in NHPs, the presence of the fovea requires particular care during intraoperative manipulation ${ }^{20}$. In particular, there is a high risk of a foveal tear if subretinal fluid injection methods generate an excessive force on the retina ${ }^{20}$. The focus of this manuscript is, therefore, on strategies to reduce the risk of inadvertent foveal trauma in NHP.

These include the use of preoperative intravitreal plasminogen injection for the release of parafoveal adhesions and surgical microscope-integrated optical coherence tomography (miOCT) intraoperatively for realtime visualization of the foveal anatomy. A custom-made 25/41 G dual-bore subretinal cannula with intraocular PFCL tamponade under low IOP settings is proposed to allow a more controlled process of foveal detachment. Furthermore, surgical removal of native RPE is recommended before implantation to allow better integration between the 
transplanted RPE cells and host photoreceptors. Finally, a peri- and postoperative systemic immunosuppression protocol for NHP models is described to improve the survival of the RPE xenograft post-transplantation 11,21 .

\section{Protocol}

NOTE: All animal experiments were conducted in accordance with The Association of Research in Vision and Ophthalmology (ARVO) for the Use of Animals in Ophthalmic and Vision Research. Ethics approval was obtained from the Institutional Animal Care and Use Committee, SingHealth, Singapore. Animals were housed at the SingHealth Experimental Medicine Centre approved by the Association for Assessment and Accreditation of Laboratory Animal Care (AAAAC). This approval highlights that all animal experiments comply with the standards of the National Advisory Committee for Laboratory Animal Research guidelines set out by the Agri-Food and Veterinary Authority of Singapore. The following experimental protocol was established based on experiments conducted in 6 eyes of 6 Macaca fascicularis (4 male and 2 female, 4 to 6 years old, 2.8 to $4.0 \mathrm{~kg}$ ) .

\section{Achieving immunosuppression in the NHP model}

1. Start the immunosuppression 7 days before surgery and continue immunosuppression throughout the follow-up period.

2. Weigh the NHP before the administration of systemic immunosuppression to ensure accurate medication dosage. The animal is weighed at baseline and weekly thereafter.

3. Use oral sirolimus, doxycycline, and minocycline to achieve systemic immunosuppression.
1. Administer a loading dose of $2 \mathrm{mg}$ of oral sirolimus followed by a daily maintenance dosing of 1 mg. Obtain baseline blood sirolimus level before administration and monitor this throughout the follow-up period. Ensure a concentration of at least $5 \mu \mathrm{g} / \mathrm{L}$ for adequate immunosuppression.

NOTE: Sirolimus dose is not weight-adapted.

2. Administer a dose of $7.5 \mathrm{mg} / \mathrm{kg}$ of oral doxycycline per day, two times per day.

3. Administer a dose of $7.5 \mathrm{mg} / \mathrm{kg}$ of oral minocycline per day, two times per day.

4. During immunosuppression, monitor all NHPs for adverse systemic effects. Look for significant body weight loss $(>10 \%)$, decreased appetite and water consumption, ungroomed hair loss, and abnormal behavior such as aggression and lethargy. Assessments will be done on Day 3, 14 and 1 month, followed by monthly assessments.

\section{Instrument sterilization}

1. Rinse the surgical instruments using distilled water.

2. Place the instruments in an ultrasonic bath filled with 500 $\mathrm{mL}$ of distilled water and $2 \mathrm{~mL}$ of instrument disinfectant. Clean the instruments using the sweep function of the ultrasonic bath for $15 \mathrm{~min}$.

3. Remove the instruments from the ultrasonic bath. Rinse twice-thoroughly-with distilled water for 5 min each rinse. Air-dry the instruments after the rinse.

4. Place the instruments in an instrument box. Autoclave the box using the universal program setting (sterilization of instruments at $134{ }^{\circ} \mathrm{C}$ for $50 \mathrm{~min}$ : $30 \mathrm{~min}$ for autoclaving, $20 \mathrm{~min}$ for drying). 


\section{Preparation of preservative-free triamcinolone (40 $\mathrm{mg} / \mathrm{mL}$ )}

1. Using a $1 \mathrm{~mL}$ syringe, withdraw $1 \mathrm{~mL}$ of triamcinolone solution (10 mg/mL). Transfer it to a $15 \mathrm{~mL}$ conical tube and mix it with $4 \mathrm{~mL}$ of sterile balanced salt solution (BSS).

2. Centrifuge the solution at $120 \times g$ for $5 \mathrm{~min}$. Ensure all the triamcinolone particles are at the bottom of the conical tube. Discard the supernatant (BSS) from the conical tube.

3. Re-suspend the triamcinolone particles with $5 \mathrm{~mL}$ of sterile BSS in the conical tube. Centrifuge the solution at $120 \times g$ for $5 \mathrm{~min}$. Discard the supernatant again.

4. Repeat step 3.3 to complete the washing of the triamcinolone particles with BSS (3x).

5. Re-suspend the triamcinolone particles with $0.25 \mathrm{~mL}$ of sterile BSS to achieve a concentration of $40 \mathrm{mg} / \mathrm{mL}$.

6. Aspirate the re-suspended triamcinolone $(40 \mathrm{mg} / \mathrm{mL})$ with a new $1 \mathrm{~mL}$ syringe. Attach a $25 \mathrm{G}$ blunt-tip flute needle, and keep the syringe with the triamcinolone solution ready for intraoperative use.

\section{Pretreatment of NHP eyes with intravitreal plasminogen $(0.25 \mu \mathrm{g} / \mu \mathrm{L})$}

1. One week before the surgery, administer an intravitreal injection $(20 \mu \mathrm{L})$ of monkey plasminogen $(0.25 \mu \mathrm{g} / \mu \mathrm{L})$.

2. Sedate the NHP before the procedure with an intramuscular injection of ketamine (10-20 mg/kg BW) and a subcutaneous injection of atropine $(0.05 \mathrm{mg} /$ $\mathrm{kg}$ BW). Administer tetracaine eyedrops for local anesthesia.
3. Before intravitreal injection, disinfect the periorbital region with $10 \%$ povidone-iodine. Disinfect the eye by administering $5 \%$ povidone-iodine to the conjunctival fornices of the NHP. Ensure the solution stays in the fornices for at least $1 \mathrm{~min}$ before rinsing thoroughly with sterile BSS.

4. Use a $250 \mu \mathrm{L}$ syringe to aspirate the prediluted monkey plasminogen $(0.25 \mu \mathrm{g} / \mu \mathrm{L})$ from the vial. Attach a $30 \mathrm{G}$ needle to the syringe, and keep the monkey plasminogen ready for intravitreal administration.

5. Use a pair of calipers to identify the injection site on the eye. Administer the intravitreal injection $3 \mathrm{~mm}$ away from the limbus.

6. Proceed with the injection with the needle directed towards the center of the globe. Upon removal of the needle from the globe, use a cotton applicator stick to tamponade the injection site and prevent the reflux of the intraocular contents.

7. Administer a lubricant gel or ointment to reduce immediate postoperative ocular surface irritation.

\section{Surgical table and equipment setup}

1. Establish a sterile field. When in the sterile field, wear surgical scrubs, mask, and hair cover at all times.

2. Prepare the preservative-free triamcinolone $(40 \mathrm{mg} / \mathrm{mL})$ for intraoperative visualization of vitreous (see section 3 ). Prepare the sterile BSS in a $10 \mathrm{~mL}$ syringe and lubricant in a $5 \mathrm{~mL}$ syringe. Place them on a drape.

3. Keep other instruments ready on a drape, including 3-0 silk, 7-0 vicryl sutures, cotton applicator sticks, wound closure strips, and chandelier endoillumination fiber wire. 
4. Connect the vitrectomy set, including the high-speed vitrector, Venturi cassette, and the $25 \mathrm{G}$ chandelier endoilluminator to the vitrectomy machine using sterile technique.

5. Open a $500 \mathrm{~mL}$ ophthalmic grade BSS bottle and connect it to the Venturi cassette according to the manufacturer's instructions. Proceed with priming the system.

6. Switch on the miOCT/surgical microscope. Select preset configurations of the surgical microscope for posterior segment surgery and illumination. Enter the details of the procedure, including ID, gender, laterality of the animal eye, and the name of the procedure.

7. Mount a non-contact, wide-angled, 128-degree fundus lens.

8. Attach sterile disposable hand-piece covers onto the surgical microscope/miOCT. Adjust the microscope position and focus using the foot pedal. Proceed with surgery.

\section{Preparation of anesthesia and positioning of the animal (preferably performed by veterinarian team)}

1. Ensure the NHP is fasted for at least $8 \mathrm{~h}$ before the induction of anesthesia to prevent regurgitation and vomiting. Sedate the NHP before the induction of anesthesia (see step 4.2 for sedation instructions).

2. Apply $1 \%$ tropicamide and $2.5 \%$ phenylephrine eyedrops at least $3 x$ with 5 min intervals to achieve pupil dilation.

3. Administer an intramuscular injection of buprenorphine (0.005-0.03 $\mathrm{mg} / \mathrm{kg}$ BW) $30 \mathrm{~min}$ before the surgery to achieve analgesia.

4. Intubate the NHP with an endotracheal tube, usually 3-5 $\mathrm{mm}$ in size. When attempting intubation, ensure that several sizes are available. Use the largest size that can be passed through the larynx without causing trauma. Measure end tidal $\mathrm{CO}_{2}$ to ensure appropriate placement of endotracheal tube.

5. Deliver $2 \%$ isofluorane gas via the endotracheal tube to induce general anesthesia. Confirm the state of general anesthesia (lack of response to touch) by assessing the NHP's response to surrounding stimuli, including sounds and touch. Use $0.5-2 \%$ isofluorane gas to maintain the state of general anesthesia.

6. Continuously monitor the NHP electrocardiogram, respiratory rate, blood pressure, and oxygen saturation during the entire surgery.

7. Position the NHP on the surgical table such that the eye is perpendicular to the surgical microscope. Administer a lubricant gel or ointment to the eye, which is not being operated on to reduce ocular surface irritation during anesthesia.

8. Cut the eyelashes using scissors to reduce the chance of infections.

9. Disinfect the periorbital region with $10 \%$ povidone-lodine. Disinfect the eye by administering $5 \%$ povidone-iodine to the conjunctival fornices of the NHP. Ensure the solution stays in the fornices for at least 1 min before rinsing thoroughly with sterile BSS.

10. Position a sterile drape such that the pre-cut opening is centered over the eye to undergo surgery. Cover the eye with an adhesive surgical incision drape.

11. Perform a lateral canthotomy on the eye to undergo surgery.

12. Insert the Lieberman speculum to ensure adequate opening of the eyelids for the visualization of the eye. 


\section{Vitrectomy}

NOTE: To access the subretinal space for the delivery of the PET-scaffold RPE graft, this protocol recommends a 4-port (valved) 25 G vitrectomy to be performed using a standard vitreoretinal surgical setup and a non-contact, wide-angled, $128^{\circ}$ fundus lens. The protocol also recommends the use of a surgical microscope equipped with miOCT to guide several critical surgical steps, including the induction of foveal detachment, implantation of the RPE graft, and subretinal fluid drainage.

1. Perform a $360^{\circ}$ conjunctival peritomy by incising the conjunctiva near the limbus using a pair of vannas scissors. Enlarge the peritomy by performing a blunt dissection.

2. Using a 25 G microvitreoretinal blade, perform a sclerotomy at 8 o'clock for the right eye or 4 o'clock for the left eye. Perform the sclerotomy $3 \mathrm{~mm}$ from the limbus of the eye.

3. Insert and suture a 25 G custom side port-infusion cannula using 7-0 vicryl suture. After confirming the intravitreal location, start the BSS infusion and set the system to maintain an IOP of $20 \mathrm{mmHg}$.

4. Using a $25 \mathrm{G}$ flat head trochar, perform a sclerotomy at 2 o'clock for the right eye or 10 o'clock for the left eye, as in step 7.2.

5. Insert the $25 \mathrm{G}$ chandelier light into the flathead trochar and secure it with sticky tape. Adjust the light source to approximately $60 \%$.

6. Perform another sclerotomy, similar to step 7.2 , at 10 o'clock for the right eye or 2 o'clock for the left eye. Place U-shaped vicryl 7-0 sutures around the sclerotomy without tying the knots. Insert the vitrectomy cutter tip through this sclerotomy.

7. Start the vitrectomy around the entry ports, followed by a short core vitrectomy with the following settings: maximum 5000 cuts per minute, maximum aspiration at $400 \mathrm{mmHg}$.

8. Inject $20-50 \mu \mathrm{L}$ of triamcinolone $(40 \mathrm{mg} / \mathrm{mL})$ for better vitreous visualization.

9. Induce a posterior vitreous detachment (PVD) by separating the vitreous body from the retina.

1. Position the vitrector above the optic disc to allow gentle induction of the PVD. Keep the vitrector only on aspiration at the maximum setting of $400 \mathrm{mmHg}$ without any cutting involved.

2. If required, use $25 \mathrm{G}$ intraocular forceps to manipulate the vitreous at the disc margin to create a tear in the vitreous cortex to facilitate detachment. NOTE: PVD is considered successful if triamcinolone crystals glide unimpeded over the retinal surface.

10. Open the posterior hyaloid membrane with the cutter, and remove the detached vitreous skirt up to the vitreous base (at the retinal equator). Aspirate any remaining triamcinolone on the retinal surface.

\section{8. miOCT-guided foveal detachment}

1. Inject 1-2 $\mathrm{mL}$ of PFCL to cover the posterior pole up to the anterior, mid-peripheral retina.

2. Enter the eye with a subretinal injection cannula. Set the IOP to $0-4 \mathrm{mmHg}$ on the vitrectomy machine (ensure perfectly watertight system; if needed, tie sutures around the ports). 
3. Using either the $25 / 41 \mathrm{G}$ customized dual-bore subretinal injection cannula or $25 / 38 \mathrm{G}$ subretinal injection cannula connected to a $250 \mu \mathrm{L}$ syringe, gently perform a subretinal injection of BSS to induce a localized retinal detachment. Once the bleb just crosses the fovea, stop the injection. Create a second bleb from a separate direction. Merge both the blebs to fully detach the fovea.

4. Enable the miOCT function to visualize bleb formation. Ensure the line and cube scans are in HD mode with the settings (512 $\times 128$ pixels, scan width $4 \mathrm{~mm}$ ) to acquire an image at the fovea. Observe the miOCT image for a complete detachment of the neural retina from the RPE layer at the fovea.

5. Enlarge the retinotomy to $1.5 \mathrm{~mm}$ with a pair of vertical 25 $G$ vitreoretinal scissors to allow access to the subretinal space for transplantation.

\section{Removal of native RPE}

1. Set the IOP to $50 \mathrm{mmHg}$ on the vitrectomy machine.

2. Remove the PFCL via active extrusion using a brushed silicone tip cannula.

3. Extend the sclerotomy with a $1.4 \mathrm{~mm}$ incision knife to allow the entrance of a $20 \mathrm{G}$ instrument.

4. Using a custom $20 \mathrm{G}$ extensible loop instrument, scrape the submacular host RPE for removal. Scrape an area that measures at least $2 \times 3 \mathrm{~mm}$.

\section{Loading of the shooter for delivery of RPE cell monolayer transplant}

1. For general instructions on the loading of a bullet-shaped graft cut from RPE cultures on PET cell carriers, refer to a previous publication ${ }^{22}$.

\section{1. miOCT-guided graft implantation and position adjustment}

1. Insert the tip of the shooter device through the sclerotomy at an IOP of $20 \mathrm{mmHg}$. Inject the implant towards the subretinal space via the retinotomy edge created from the retinal surface.

2. Inject the implant with the cell carrier side facing the Bruch's membrane and the RPE xenograft side facing the photoreceptors.

3. Enable the miOCT function to visualize the implant location. Ensure the implant is resting flat on the Bruch's membrane in the subretinal space, with an intact overlying retina. Ensure it is located a reasonable distance away from the created retinotomy and not impinged on the retinotomy site.

4. Adjust the implant position with the subretinal injection cannula or a $25 \mathrm{G}$ curved intraocular scissor to ensure it is well-positioned under the macula.

\section{2. miOCT-guided drainage of subretinal fluid}

1. Using a brushed silicone tip cannula, perform a fluid-air exchange and careful subretinal fluid drainage. Attempt gentle subretinal fluid aspiration from the bleb retinal detachment and retinotomy edge apposition.

2. Enable the miOCT function for real-time visualization of adequate subretinal fluid drainage until the retina is reattached over the implant.

\section{Ending the operation}

1. Close the working port sclerotomy using the preplaced 7-0 vicryl suture. Remove the $25 \mathrm{G}$ chandelier and $25 \mathrm{G}$ 
infusion cannula. Close these sclerotomies with 7-0 vicryl sutures.

2. Administer $2 \mathrm{mg}$ in $0.05 \mathrm{ml}$ of intravitreal preservative free triamcinolone $(40 \mathrm{mg} / \mathrm{ml})$ at the 8 o'clock sclerotomy prior to suturing.

3. Palpate the eye to ensure that the IOP is within the acceptable range. Inject filtered air (or BSS) via a $30 \mathrm{G}$ needle if required.

4. Suture the conjunctiva with 7-0 vicryl sutures and canthotomy with 5-0 prolene (remove after 10-14 days).

\section{Postoperative animal care}

1. Position the NHP face down for $1 \mathrm{~h}$ post-surgery. Do not leave the animal unattended until consciousness has been regained. Ensure that a veterinarian and animal care technician are available for observation and support during the postoperative process.

2. Apply topical antibiotic (tobramycin), steroid (dexamethasone) ointment, and homatropine eye drops twice a day for 5 days postoperatively.

3. Administer another subcutaneous buprenorphine (0.005-0.03 mg/kg BW) injection $6 \mathrm{~h}$ after surgery for adequate pain control.

4. Return the NHP to the company of other animals only when it has fully regained consciousness.

5. Conduct multimodal imaging follow-ups on Day 3, 14 and Month 1 after the procedure, followed by monthly checkups. Perform ERGs every month after the procedure. Remove the 5-0 prolene sutures for the canthotomy at Day 14 concurrently with the sedation period used for multimodal imaging. The remaining sutures are resorbable, 7-0 vicryl sutures, which do not require removal.

\section{Postoperative monitoring methods for multimodal imaging}

1. Fast the NHP overnight. Sedate the NHP just before imaging (see step 4.2 for drug and concentration for sedation). If sedation is insufficient to stop eye movement, consider the use of general anesthesia.

2. Apply $1 \%$ tropicamide and $2.5 \%$ phenylephrine eyedrops to achieve pupil dilation before imaging (see step 6.2).

3. Perform autofluorescence (AF), fundus fluorescein angiography (FFA), and optical coherence tomography (OCT) using a high-resolution OCT machine with a $55^{\circ}$ field lens and $30^{\circ}$ field lens.

1. Administer intravenous $10 \%$ fluorescein $(0.1 \mathrm{~mL} /$ $\mathrm{kg} \mathrm{BW)} \mathrm{for} \mathrm{FFA.} \mathrm{To} \mathrm{obtain} \mathrm{an} \mathrm{early} \mathrm{phase} \mathrm{image,}$ capture an image within $30 \mathrm{~s}$ of injection. For a late phase image, capture an image 5-10 min after the injection.

4. Perform fundus photography using a fundus camera between the early and late phases of FFA.

\section{Postoperative monitoring methods for full-field electroretinogram (ERG) studies}

1. Fast the NHPs overnight. Sedate the NHP before ERG studies (see step 4.2 for drug and concentration for sedation). Throughout the ERG recordings, readminister sedation when appropriate.

2. Separate multimodal imaging and ERG recordings with an interval of at least 2-3 days.

3. Once sedated, ensure the NHP is dark-adapted for 30 min before the ERG recording.

4. Position the stainless-steel subdermal needle electrodes at the left and right lateral canthi (reference electrodes) 
and the back of the NHP body (ground electrode). Place the ERG contact lens electrodes on the NHP cornea using vidisic gel to aid contact and adhesion.

5. Base all ERG testing on the human protocols recommended by the International Society for Clinical Electrophysiology of Vision (ISCEV) ${ }^{14}$. Commence ERG recording under scotopic conditions and start with the dimmer flashes. Follow the ISCEV recommendations for recommended interstimulus intervals.

6. Ensure the NHP is light-adapted for $10 \mathrm{~min}$ before photopic testing, again using standard ISCEV recommendations for background strength.

\section{Euthanasia of NHP}

1. To euthanize the NHP for enucleation, administer intravenous sodium pentobarbital (75 mg/kg), as recommended by the Panel on Euthanasia of the American Veterinary Medical Association.

\section{Representative Results}

The multimodal imaging modalities (fundus photography, fundus autofluorescence imaging (FAF), fundus fluorescein angiography (FFA)-early-phase and late-phase, and optical coherence tomography (OCT)) highlight the features of a successful submacular RPE graft transplant (Figure 1). Fundus photography shows the positioning of the RPE graft transplant at the fovea without migration over time. FAF imaging shows minimal changes in hyper-autofluorescence (demonstrated by white, high-intensity areas) overlapping the RPE graft. Early- and late-phase FFA do not show any obvious leakage (demonstrated by white, high-intensity areas that enlarge with time) surrounding the RPE graft. Initial images at Day 3 show window defect due to the removal of native RPE before graft implantation. Macular
OCT images show the preservation of outer retinal layers (in particular, the photoreceptor layer) over the RPE graft as time progresses. Hematoxylin and eosin staining shows intact retinal layers with no evidence of microtears. The preservation of the outer nuclear layer above the peripheries of the graft suggests that the RPE cells are performing their physiological functions of maintaining photoreceptor health.

The intraocular and external views of the 25/41 G dualbore cannula highlight the mechanism by which the IOP is controlled during subretinal injection (Figure 2). BSS enters the subretinal space during subretinal fluid injection via the central longer cannula. Significant increases in intraocular pressure cause the BSS within the vitreous cavity to exit the eye via the larger metal bore of the cannula. BSS then travels along the cannula and is eventually ejected from the egress port near the cannula hub. To assess whether the cannula is working as expected, ensure that fluid flows from the egress port near the cannula hub.

The miOCT allows the visualization of the bleb dimensions and a potential foveal tear intraoperatively during foveal detachment (Figure 3). Figure 3A1-A3 highlight a case of bleb with a foveal tear. In Figure 3A1, while the inferior bleb is visible under the surgical microscope, visualization of the tear is difficult. Figure $3 \mathbf{A} 2$ shows the longitudinal section of a bleb without any tears. Figure $\mathbf{3 A} \mathbf{3}$ shows a foveal tear when assessing the vertical section of the bleb. Figure 3B1-B3 show a successfully created bleb without the presence of any tears.

The absence of significant deterioration in the ERG waveforms suggests that the global function of both rod and cone photoreceptors is maintained with subretinal RPE xenografts (Figure 4). The ERG waveforms show the overall 
function of the retina. In particular, attention should be paid to

the A-waves to determine any loss of photoreceptor function.

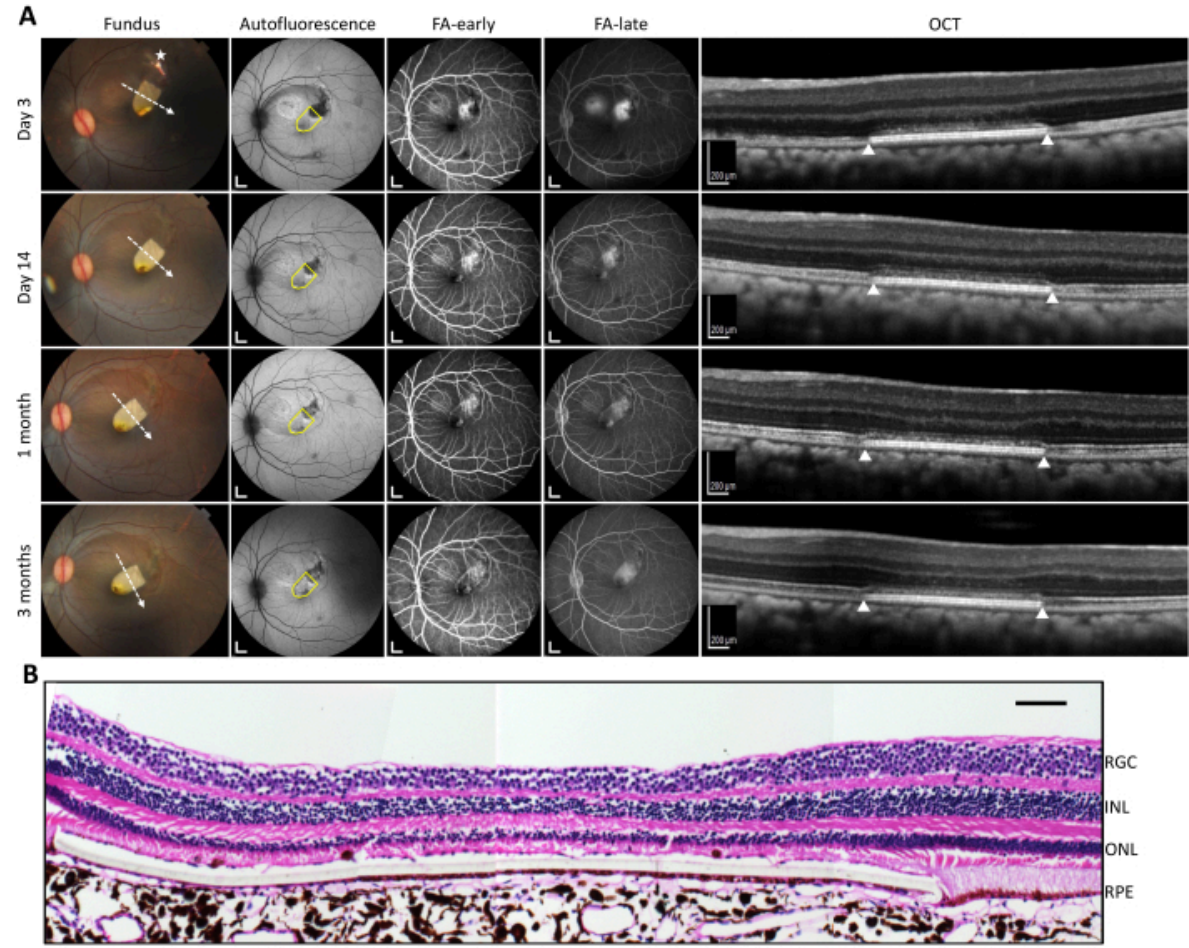

Figure 1: Postoperative in vivo analysis with multimodal imaging. (A) In vivo imaging of the left eye submacular RPE graft transplant (yellow on fundus photography) on various imaging modalities (left to right columns: fundus photography, autofluorescence, fundus fluorescein angiography-early phase, fundus fluorescein angiography-late phase, optical coherence tomography) for time points up to 3 months (top to bottom rows: Days 3,14 ; Months 1,3 ). The asterisk on the fundus photograph indicates the site of the retinotomy; the white dashed arrow indicates the direction of the line scan. The yellow drawn shape on fundus autofluorescence imaging highlights the location of the transplant. The white triangles on the OCT images indicate the respective lateral edges of the graft (as per the line scan on the color fundus image). (B) Hematoxylin and eosin staining of the transplant under atrophic fovea (due to intraoperative tear) with layers labeled. Scale bars = $1 \mathrm{~mm}$ in $\mathbf{A}$ (autofluorescence and FA images), $200 \mu \mathrm{m}$ in $\mathbf{A}$ (OCT images), and $100 \mu \mathrm{m}$ in $\mathbf{B}$. Abbreviations: FA = fundus angiography; OCT = optical coherence tomography; RGC = retinal ganglion cell layer; INL = inner nuclear layer; ONL $=$ outer nuclear layer; RPE = retinal pigment epithelium. Please click here to view a larger version of this figure. 


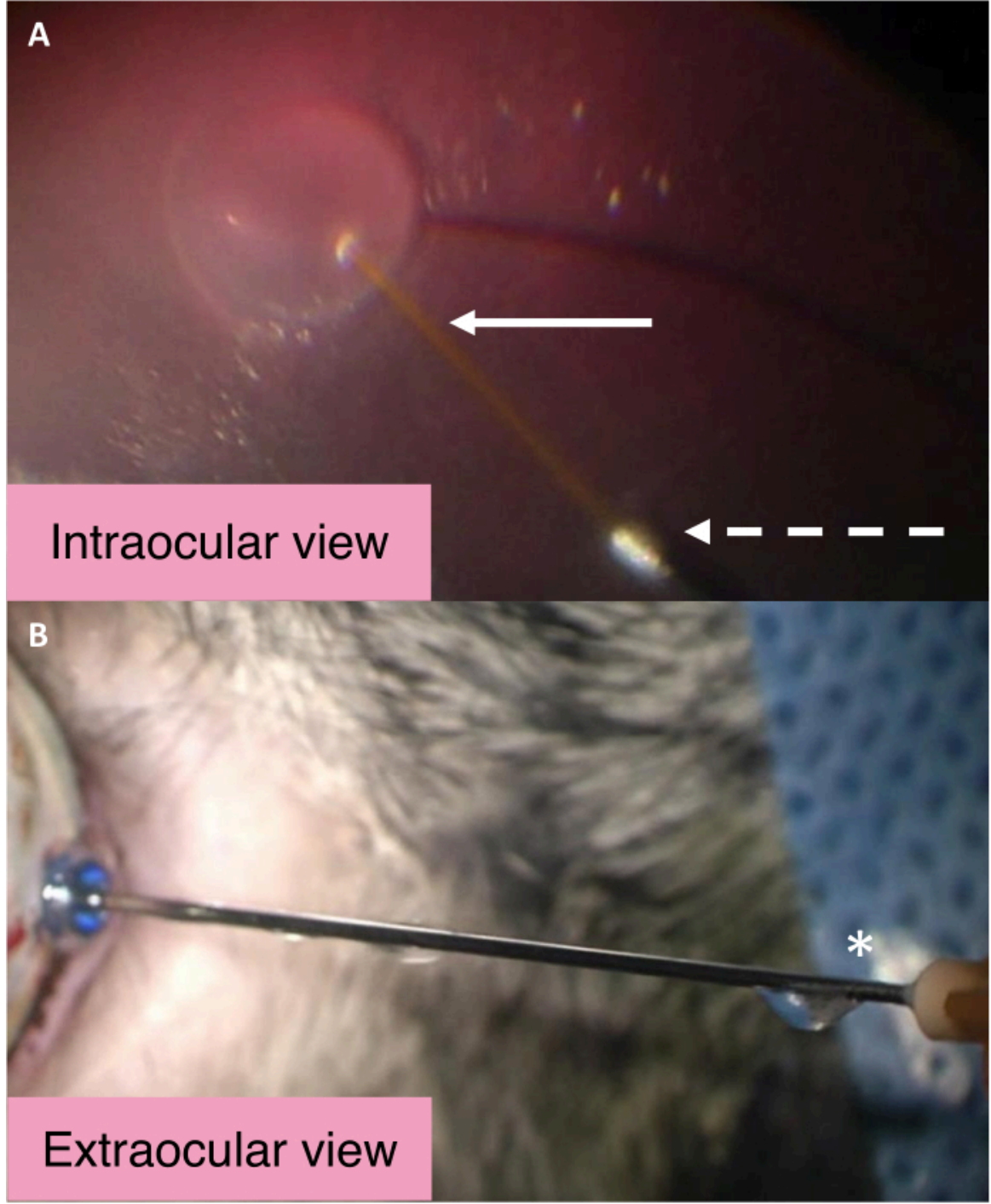

Figure 2: Intraocular and external views of the 25/41 G dual-bore cannula. (A) Intraocular view of the 25/41 G dual-bore cannula during subretinal bleb creation. The white arrow points to the longer central cannula for subretinal injection. The dashed arrow points to the opening of the egress cannula through which the BSS passes to exit the eye. (B) External view of the 25/41 G dual-bore cannula. The asterisk marks the egress port near the cannula hub from which the intraocular BSS is drained. Abbreviation: BSS = balanced salt solution. Please click here to view a larger version of this figure. 

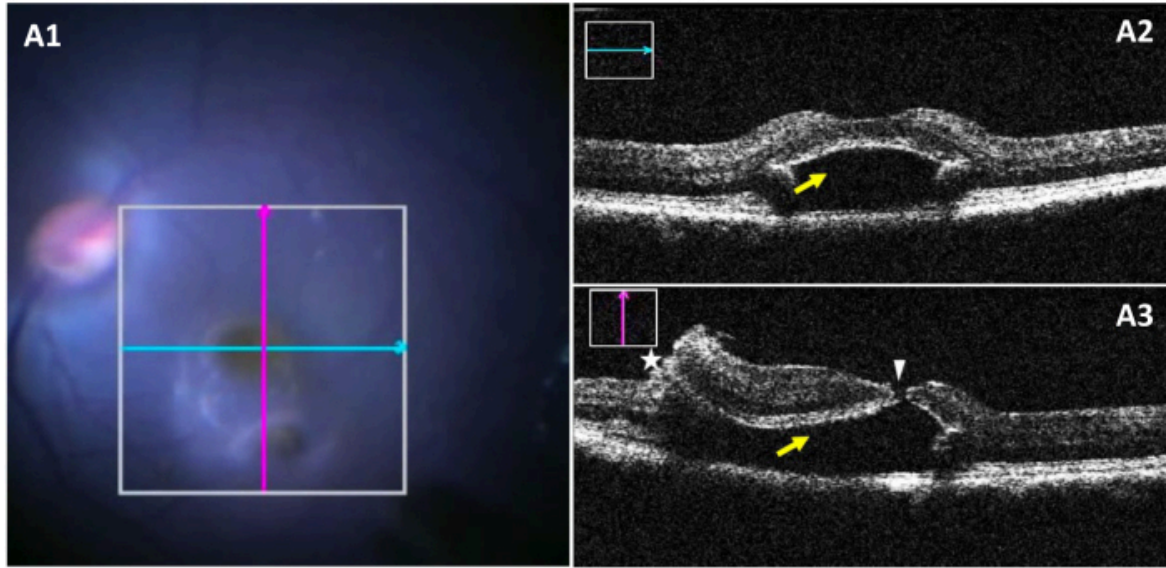

B1
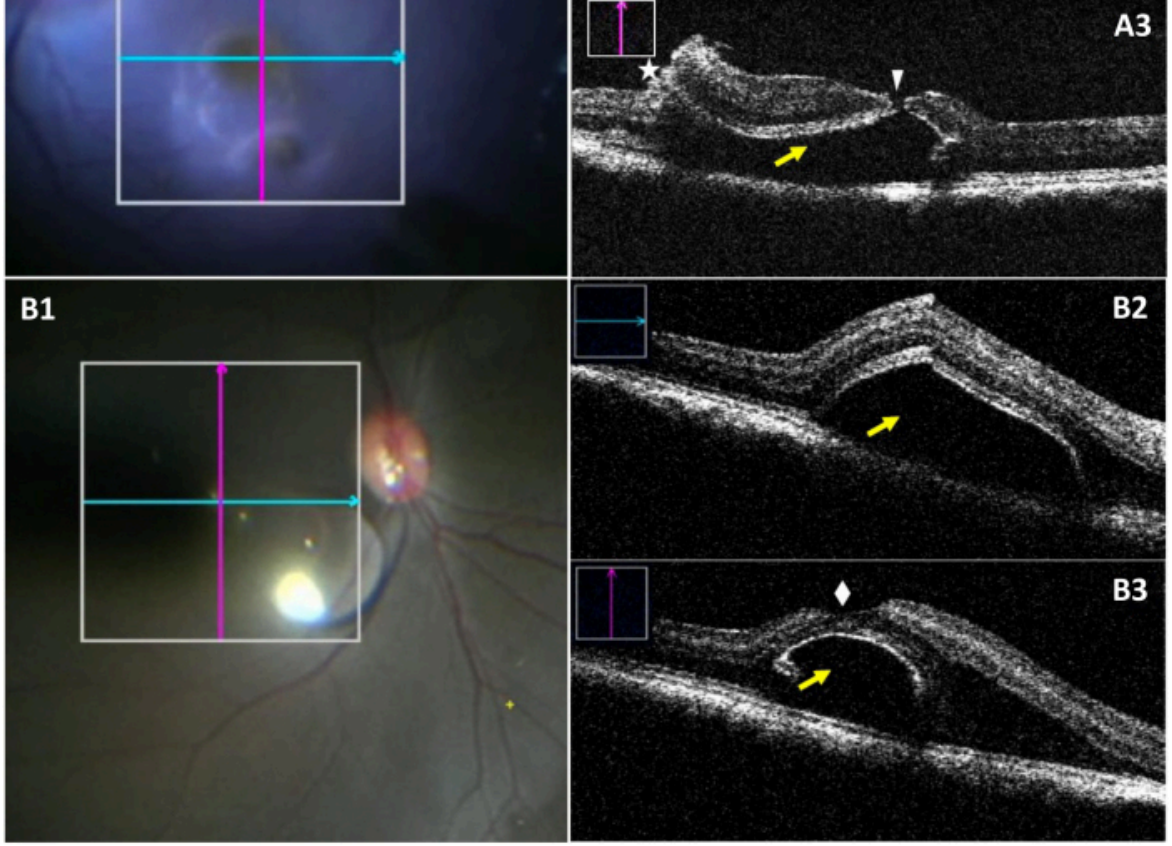

Figure 3: Intraoperative microscope images and miOCT images of subretinal bleb complicated by a foveal tear. (A1) An intraoperative microscope image showing the position of longitudinal (blue) and transverse (red) scans in a bleb with a foveal tear. (A2) Longitudinal miOCT scan showing a subretinal bleb at the foveal region (yellow arrow). (A3) Transverse miOCT scan capturing a foveal tear (white arrowhead), along with a retinotomy (asterisk and a subretinal bleb (yellow arrow). (B1) An intraoperative microscopic image showing the position of longitudinal (blue) and transverse (red) scans in a successfully formed bleb. (B2) Longitudinal miOCT scan showing a subretinal bleb at the foveal region (yellow arrow). (B3) Transverse miOCT scan showing a successfully created subretinal bleb with an intact fovea superiorly (white diamond). Abbreviation: $\mathrm{miOCT}=$ microscope-integrated optical coherence tomography. Please click here to view a larger version of this figure. 
RPE xenograft transplanted eye
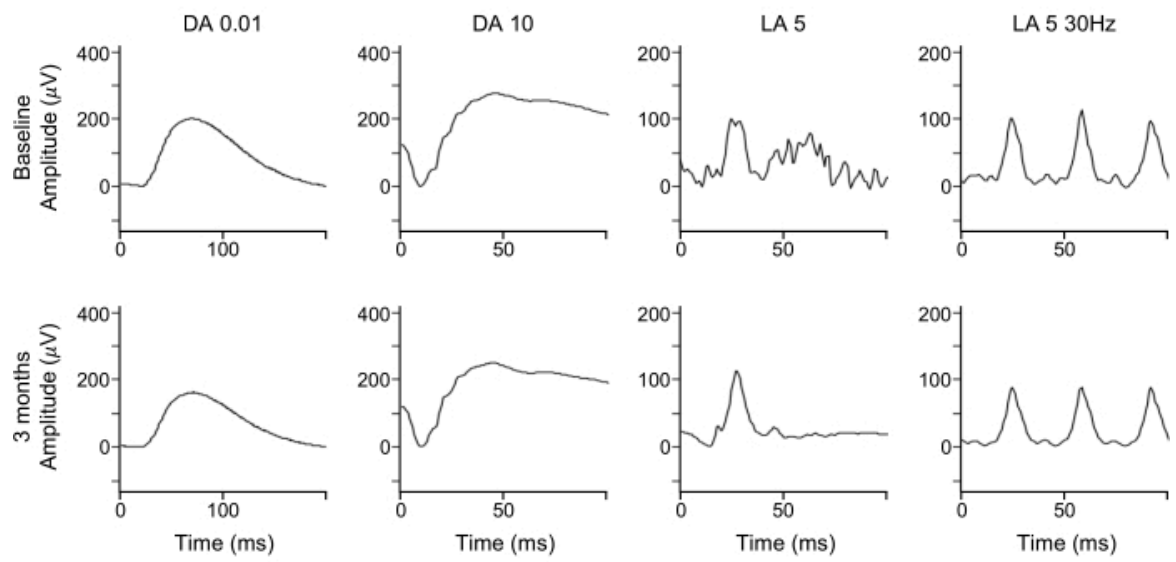

Figure 4: ERG of RPE xenograft-transplanted eye. For the functional assessment of the retina, full-field ERG assessments of the RPE-xenografted eye performed at baseline (top row) and 3 months post-transplantation (bottom row) show no significant effect of the RPE xenograft transplantation on any response amplitudes, timing, or waveform under either dark-adapted or light-adapted conditions. Abbreviations: RPE = retinal pigment epithelium; $E R G=$ electroretinogram; DA = dark-adapted; LA = light-adapted. Please click here to view a larger version of this figure.

\section{Discussion}

There are two main approaches being evaluated for submacular RPE transplantation-the injection of an RPE suspension and the transplantation of a monolayer RPE graft. A detailed comparison between the two methods is beyond the scope of this manuscript. However, the transplantation of a monolayer RPE graft may be advantageous as the RPE cells are more organized in a monolayer than in a suspension. RPE cells in the graft are organized in a confluent monolayer, which resembles the organization of the physiological RPE cell layer and enables the transplanted RPE cells to perform their physiological functions. This enables more precise dosing parameters compared to cell suspensions, which is highly relevant for regulatory work and industrial scale-up.
Delivery of the RPE patch graft into the subretinal space requires careful manipulation of the macula and accurate insertion of the graft in the subretinal space. Technological advances in microsurgery, such as miOCT, and a better understanding of intraoperative retinal tissue dynamics have reduced the learning curve of this procedure. In this discussion, the rationales of the following aspects will be explained: i) pre-operative plasminogen injection; ii) the use of intraoperative miOCT; iii) the use of a custom $41 \mathrm{G}$ dualbore cannula, low IOP settings, and PFCL for subretinal bleb creation; iv) scraping of the native RPE cell layer before transplantation; v) the use of sirolimus, triamcinolone, doxycycline, and minocycline to reduce immunogenic graft rejection. 
Preoperative plasminogen injections release parafoveal retinal adhesions

In the initial experiments, it was challenging to detach the fovea with a single fluid wave. On assessment with miOCT, the images revealed the presence of parafoveal outer retinal adhesions to the native RPE along with evidence of intraretinal trauma ${ }^{20}$. These adhesions may have led to a vertical expansion of the bleb rather than the subretinal fluid wave spreading across the retinal contour, resulting in foveal trauma. Plasminogen is the inactive precursor of plasmin, a protease targeting fibronectin and laminin. Ocriplasmin is a bioengineered variant of human plasmin, approved by the Food and Drug Administration (FDA) and European Medicines Agency (EMA) for the treatment of symptomatic vitreomacular traction with or without a concomitant macular hole. However, postapproval reports of cystoid macula edema development after ocriplasmin injection have suggested a more extensive effect of the enzyme on the retina ${ }^{23}$.

Although the exact mechanisms have not been identified, it was suggested that plasmin could weaken retinal adhesion through the degradation of the interphotoreceptor matrix elements responsible for photoreceptor-RPE adhesion ${ }^{24}$. In this protocol, NHP eyes were treated with intravitreal plasminogen 1 week before surgery to release the parafoveal outer retinal adhesions. Under the assumption that the photoreceptor-RPE adhesion is weakened, a lower force is required to detach the neurosensory retina, including the distal parafoveal ring, which typically resists the subretinal fluid wave $\mathrm{e}^{20}$. Thus, the force administered during retinal bleb detachment results in the expansion of the bleb across the retinal contour rather than stretching the retina tangentially. This reduces the risk of foveal tears. However, it should be noted that the effect of plasminogen on long-term graft survival was not studied in this protocol. Future studies should attempt to determine this effect.

miOCT provides anatomical feedback to guide subretinal bleb creation, graft implantation, and subretinal fluid drainage

Intraoperative, atraumatic manipulation of the macula is key to achieving good transplantation outcomes. However, microstructural changes of the macula related to manipulation may not always be evident on the operating microscope. In such procedures, the miOCT is an important tool that provides real-time, three-dimensional, intraoperative feedback of the macular structure. miOCT is especially useful during the steps of foveal detachment, graft implantation, and drainage of the subretinal fluid using a fluid-air exchange. During foveal detachment, miOCT can determine the vertical and horizontal dimensions of the bleb. Foveal microtears, which may not be visualized clearly on the surgical microscope, can be confirmed by miOCT (Figure 3). During the graft implantation, miOCT images guide by showing the graft's location or proximity to the fovea, through the often less-transparent, detached retina. miOCT can also highlight possible areas of retinal adhesion during a difficult transplantation process ${ }^{25}$. Finally, in the subretinal fluid drainage process, miOCT can reliably guide subretinal fluid drainage until complete retinalRPE graft contact is achieved.

The combination of a dual-bore cannula, low IOP settings, and PFCL vitreous tamponade synergistically reduces macular trauma during subretinal bleb creation Tangential retinal stretching and fluid turbulence can occur during the subretinal BSS injection for foveal detachment leading to unwanted foveal tears. To counteract these phenomena, factors, such as the relative position and distance from the foveal center where the injection is initiated, 
injection volume and velocity, vitreous tamponade, choice of subretinal instrumentation, and IOP have all been shown to be relevant $^{20,26,27}$. The subretinal bleb for foveal detachment should be situated at a location adequately distant from the fovea, as retinal stretching may be highest at the bleb initiation site $^{27}$. IOP should also be kept low throughout the creation of the subretinal bleb. When the IOP of the eye is high, a higher vertical increase in bleb size rather than expansion along the contour of the retina is observed, whereas blebs are shallower at lower pressures ${ }^{20}$. Furthermore, although an intravitreal injection of $50 \mu \mathrm{L}$ will effectively double the IOP in humans $^{28}$, given the shorter eye length in NHPs, the IOP rise during subretinal injection will probably be higher and more rapid than in humans. While most vitrectomy machines adjust for IOP fluctuation, the adjustment is not a simultaneous but rather a reactive process that occurs as subretinal injection proceeds. Hence, the higher the IOP, the higher the risk of retinal overstretching and resultant foveal trauma. Thus, it is essential to maintain a stable low IOP during subretinal injection.

A commercial 20/41 G (DORC) or a custom-made 25/41 G dual-bore subretinal cannula is recommended for subretinal injection. The cannula allows fluid to exit the vitreous cavity in exchange for BSS injected into the subretinal space. This ensures the 'simultaneous' regulation of IOP during the subretinal injection. A schematic of the dual-bore cannula is seen in Figure 2. Finally, PFCL is utilized to reduce the risk of foveal tears $20,26,27$. As PFCLs, such as octaline, have higher specific gravity, they exert a downward force on the retina during foveal detachment ${ }^{29}$. This further stabilizes the foveal detachment bleb creation process and enhances the expansion of the bleb along the retinal contour. This technique has been successfully used for the subretinal injection of
rtPA in the setting of massive submacular hemorrhage due to $\mathrm{nAMD}{ }^{30}$.

\section{Pretransplantation removal of native RPE allows the} restoration of RPE-photoreceptor complex

Host RPE should be removed before graft transplantation. This is because the restoration of the RPE-photoreceptor complex is required to enable the RPE transplant to perform its physiological functions of supporting the photoreceptors ${ }^{21}$. The host RPE, if not removed, may pose as a mechanical barrier, which prevents the restoration of this complex. It can be removed either through the administration of RPEtoxic chemicals or by using physical means of removal. Chemical removal methods include the systemic or subretinal administration of sodium iodate ${ }^{31,32}$. As sodium iodate causes widespread degeneration of photoreceptors, RPE cells, and Choriocapillaris when administered, its retinal and systemic toxicity precludes its use for human trials ${ }^{32,33}$. Hence, physical intraoperative techniques are preferred. Various physical methods have been conceptualized. When physical methods are utilized, it is crucial that the Bruch's membrane remains undamaged. Many in vitro studies have demonstrated the dependence of RPE graft survival on an intact Bruch's membrane $34,35,36$.

Attempts at hydraulic debridement were associated with breaks in Bruch's membrane, an increased rate of epiretinal membrane development, and proliferative vitreoretinopathy, resulting in tractional retinal detachment ${ }^{37}$. A diamonddusted spatula proposed for RPE debridement also led to breaks in the Bruch's membrane, resulting in cellular proliferation from the choroid into the subretinal space ${ }^{38}$. Interestingly, a custom-made extendable loop instrument could remove the overlying RPE with preservation of Bruch's membrane in the eyes of rabbits and pigs ${ }^{11,39}$. The removal 
of the underlying RPE is also useful for establishing animal models with RPE and outer retinal atrophy, similar to the advanced atrophic form of AMD. When a focal area of RPE is removed from the macula, the RPE wound closes via the hypertrophy of the remaining RPE cells. However, this wound healing response is associated with atrophy of the outer nuclear layer ${ }^{40}$. While the creation of an animal model is beyond the scope of this manuscript, a similar procedure can create an animal model of an advanced atrophic AMD phenotype for the testing of RPE-derived cell therapeutics.

The use of sirolimus, triamcinolone, doxycycline, and minocycline to reduce immunogenic graft rejection

The subretinal space is thought to be an immune-privileged site, maintained by an intact blood-retinal barrier and other factors ${ }^{41}$. In many studies involving the subretinal transplantation of stem-cell derivatives with an intact bloodretinal barrier, immunosuppressive drugs play a negligible role in graft survival ${ }^{42}$. The outer blood-retinal barrier is thought to be formed by the native RPE layer and the tight junctions between the RPE cells. While native RPE removal allows better integration of the transplanted RPE and host photoreceptors, the blood-retinal barrier is disrupted in the process, increasing the likelihood of an immune rejection. Classically, T-cells are central to the process of transplant rejection of other organs such as the kidney and liver ${ }^{43}$. Hence, initial immunosuppressive regimens for retinal tissue transplantation were targeted towards reducing these adaptive immune responses.

Sirolimus, a mechanistic target of rapamycin inhibitor, and tacrolimus, a calcineurin inhibitor, are examples of immunosuppressive drugs targeting adaptive immune responses. However, despite adequate T-cell suppression, graft survival rates remain low. In addition, RPE cells are known to suppress T-cell activation through the release of inhibitory factors and promote the generation of regulatory $\mathrm{T}_{\text {-cells }}{ }^{44}$. Hence, it has become increasingly apparent that adaptive immunity may not be the only contributor to graft rejection ${ }^{42}$. Subretinal transplantation of cellular products can result in the accumulation and activation of microglia ${ }^{45}$.

Microglia are the macrophages of the retina. They consist of two main populations: 1) the perivascular microglia of the inner retinal vasculature and 2) the microglia within the retinal tissue parenchyma. As microglia are part of the innate immune response, intravitreal glucocorticoids, such as triamcinolone, can suppress cytokine-mediated proliferation ${ }^{46}$. Doxycycline and minocycline can also suppress microglial activation and should be considered ${ }^{47,48}$. Lastly, differences in immune rejection of RPE allografts versus xenografts are incompletely understood $^{49}$. For instance, alloantibodies against induced pluripotent stem cell-derived RPE cells have been reported in the serum of in vivo immune rejection models. However, the role of these antibodies and the importance of antibodymediated rejection in graft survival remains unknown ${ }^{50}$. Hence, a multidrug regimen utilizing sirolimus for the suppression of adaptive immunity and a combination of triamcinolone, doxycycline, and minocycline for innate immunity suppression is proposed. This regimen has been successfully used in rabbits with good graft survival outcomes and minimal systemic effects ${ }^{11}$.

\section{Limitations of this surgical technique}

This paper describes a possible surgical method to deliver an RPE graft sheet into the subretinal space of NHP; however, this does not mean this is the only optimized way. Different vitreo-retinal surgeons may have other preferences for instrumentation and technique. For example, this implantation 
device design can only deliver flat implants supported with a stiffer cell carrier and hence may not be suitable for relatively flexible (or rolled) implants. RPE suspension transplants can omit much of this technique. Accordingly, surgical details will require modification based on each delivery strategy.

As interest in cellular therapeutics for the treatment of degenerative retinal diseases continues to grow, the NHP animal model will be essential in preclinical studies for studying the factors affecting RPE graft survival. In this manuscript, strategies are proposed to enable the smoother delivery of a submacular monolayer RPE graft in the NHP eye. Methods for better visualization of intraoperative complications are also recommended. It is anticipated that these methods will continue to improve as the use of cellular therapeutics expands. Future method papers should also consider proposing a comprehensive list of investigations to assess various structural and functional aspects of the graft.

\section{Disclosures}

Boris Stanzel holds a US Patent 9980851 on an instrument (RPE scraper) used in this study. Speaker honoraria from C. Zeiss Meditec and Geuder to Boris Stanzel. The other authors have no conflict of interests to declare.

\section{Acknowledgments}

This study was supported by IAF-PP (HMBS Domain) (OrBID): OculaR Blomaterials and Device, A*STAR, Singapore (H17/01/a0/013), the NUS Start-up grant NUHSRO/2016/100/SU/01, NUHS Clinical Scientist Program (NCSP) grant and National Research Foundation Competitive Research Programme, Singapore (NRF-CRP21-2018-0008) to X.S., Hong Leong Endowed Professorship funds to G.E.H. and B.V.S. We would like to acknowledge the veterinary team at the Translational Pre-Clinical Model Platform (Singapore
Eye Research Institute, Singapore) for providing support in NHP surgery preparation and animal follow-up. We would like to extend our appreciation to Jill Teo and colleagues from C. Zeiss Meditec Singapore for technical support for the OPMILumera 700 with integrated intraoperative OCT device.

\section{References}

1. Wong, W. L. et al. Global prevalence of age-related macular degeneration and disease burden projection for 2020 and 2040: a systematic review and meta-analysis. Lancet. Global Health. 2 (2), e106-116 (2014).

2. Verbakel, S. K. et al. Non-syndromic retinitis pigmentosa. Progress in Retinal and Eye Research. 66, 157-186 (2018).

3. Schwartz, S. D. et al. Human embryonic stem cellderived retinal pigment epithelium in patients with agerelated macular degeneration and Stargardt's macular dystrophy: follow-up of two open-label phase 1/2 studies. Lancet. 385 (9967), 509-516 (2015).

4. Kashani, A. H. et al. A bioengineered retinal pigment epithelial monolayer for advanced, dry age-related macular degeneration. Science Translational Medicine. 10 (435), eaao4097 (2018).

5. da Cruz, L. et al. Phase 1 clinical study of an embryonic stem cell-derived retinal pigment epithelium patch in agerelated macular degeneration. Nature Biotechnology. 36 (4), 328-337 (2018).

6. Mehat, M. S. et al. Transplantation of human embryonic stem cell-derived retinal pigment epithelial cells in macular degeneration. Ophthalmology. 125 (11), 1765-1775 (2018). 
7. Mandai, M. et al. Autologous induced stem-cell-derived retinal cells for macular degeneration. New England Journal of Medicine. 376 (11), 1038-1046 (2017).

8. Sugita, S. et al. HLA-matched allogeneic iPS cellsderived RPE transplantation for macular degeneration. Journal of Clinical Medicine. 9 (7), 2217 (2020).

9. Gouras, P., Flood, M. T., Kjeldbye, H. Transplantation of cultured human retinal cells to monkey retina. Anais da Academia Brasileira de Ciências. 56 (4), 431-443 (1984).

10. Koster, C. et al. A systematic review on transplantation studies of the retinal pigment epithelium in animal models. International Journal of Molecular Sciences. 21 (8), 2719 (2020).

11. Stanzel, B. et al. Surgical approaches for cell therapeutics delivery to the retinal pigment epithelium and retina. Advances in Experimental Medicine and Biology. 1186, 141-170 (2019).

12. Kamao, $\mathrm{H}$. et al. Characterization of human induced pluripotent stem cell-derived retinal pigment epithelium cell sheets aiming for clinical application. Stem Cell Reports. 2 (2), 205-218 (2014).

13. Ben M'Barek, K. et al. Clinical-grade production and safe delivery of human ESC derived RPE sheets in primates and rodents. Biomaterials. 230, 119603 (2020).

14. Fujii, S. et al. A strategy for personalized treatment of iPS-retinal immune rejections assessed in cynomolgus monkey models. International Journal of Molecular Sciences. 21 (9), 3077 (2020).

15. Kolb, H., Nelson, R., Ahnelt, P., Ortuño-Lizarán, I., Cuenca, N. The Architecture Of The Human Fovea. Webvision. Moran Eye Center. https:// webvision.med.utah.edu/book/part-ii-anatomy-and- physiology-of-the-retina/the-architecture-of-the-humanfovea/. (2021).

16. Francis, P. J. et al. Rhesus monkeys and humans share common susceptibility genes for age-related macular disease. Human Molecular Genetics. 17 (17), 2673-2680 (2008).

17. Picaud, S. et al. The primate model for understanding and restoring vision. Proceedings of the National Academy of Sciences of the United States of America. 116 (52), 26280-26287 (2019).

18. Pennesi, M. E., Neuringer, M., Courtney, R. J. Animal models of age related macular degeneration. Molecular Aspects of Medicine. 33 (4), 487-509 (2012).

19. Al-Nawaiseh, S. et al. A step by step protocol for subretinal surgery in rabbits. Journal of Visualized Experiments: JoVE. (115), 53927 (2016).

20. Tan, G. S. W. et al. Hints for gentle submacular injection in non-human primates based on intraoperative OCT guidance. Translational Vision Science \& Technology. 10 (1), 10 (2021).

21. Liu, Z. et al. Surgical transplantation of human RPE stem cell-derived RPE monolayers into non-human primates with immunosuppression. Stem Cell Reports. 16 (2), 237-251 (2021).

22. Stanzel, B. V. et al. Human RPE stem cells grown into polarized RPE monolayers on a polyester matrix are maintained after grafting into rabbit subretinal space. Stem Cell Reports. 2 (1), 64-77 (2014).

23. Shaikh, M., Miller, J. B., Papakostas, T. D., Husain, D. The efficacy and safety profile of ocriplasmin in vitreomacular interface disorders. Seminars in Ophthalmology. 32 (1), 52-55 (2017). 
24. Johnson, M. W., Fahim, A. T., Rao, R. C. Acute ocriplasmin retinopathy. Retina. 35 (6), 1055-1058 (2015).

25. Kashani, A. H. et al. Surgical method for implantation of a biosynthetic retinal pigment epithelium monolayer for geographic atrophy: experience from a phase 1/2a study. Ophthalmology. Retina. 4 (3), 264-273 (2020).

26. Maguire, A. M. et al. Safety and efficacy of gene transfer for Leber's congenital amaurosis. New England Journal of Medicine. 358 (21), 2240-2248 (2008).

27. Xue, K., Groppe, M., Salvetti, A. P., MacLaren, R. E. Technique of retinal gene therapy: delivery of viral vector into the subretinal space. Eye. 31 (9), 1308-1316 (2017).

28. Grzybowski, A. et al. 2018 Update on intravitreal injections: Euretina Expert Consensus Recommendations. Ophthalmologica. 239 (4), 181-193 (2018).

29. Wong, D., Williams, R., Stappler, T., Groenewald, C. What pressure is exerted on the retina by heavy tamponade agents? Graefe's Archive for Clinical and Experimental Ophthalmology. 243 (5), 474-477 (2005).

30. Steel, G. B., Kearns, V., Stanzel, B.V., Wong, D. Subretinal injection under perfluorocarbon liquids to avoid foveal dehiscence. Retina. (2021).

31. Petrus-Reurer, S. et al. Integration of subretinal suspension transplants of human embryonic stem cellderived retinal pigment epithelial cells in a largeeyed model of geographic atrophy. Investigative Ophthalmology \& Visual Science. 58 (2), 1314-1322 (2017).

32. Koh, A. E.-H. et al. Retinal degeneration rat model: A study on the structural and functional changes in the retina following injection of sodium iodate. Journal of Photochemistry and Photobiology B: Biology. 196, 111514, (2019).

33. Bürgi, H., Schaffner, T. H., Seiler, J. P. The toxicology of iodate: a review of the literature. Thyroid. 11 (5), 449-456 (2001).

34. Tezel, T. H., Kaplan, H. J., Del Priore, L. V. Fate of human retinal pigment epithelial cells seeded onto layers of human Bruch's membrane. Investigative Ophthalmology \& Visual Science. 40 (2), 467-476 (1999).

35. Tezel, T. H., Del Priore, L. V. Reattachment to a substrate prevents apoptosis of human retinal pigment epithelium. Graefe's Archive for Clinical and Experimental Ophthalmology. 235 (1), 41-47 (1997).

36. Castellarin, A. A. et al. In vitro transplantation of fetal human retinal pigment epithelial cells onto human cadaver Bruch's membrane. Experimental Eye Research. 66 (1), 49-67 (1998).

37. Lopez, P. F. et al. Retinal pigment epithelial wound healing in vivo. Archives of Ophthalmology. 113 (11), 1437-1446 (1995)

38. Lopez, R., Gouras, P., Brittis, M., Kjeldbye, H. Transplantation of cultured rabbit retinal epithelium to rabbit retina using a closed-eye method. Investigative Ophthalmology \& Visual Science. 28 (7), 1131-1137 (1987).

39. Thieltges, F. et al. Localized RPE removal with a novel instrument aided by viscoelastics in rabbits. Translational Vision Science \& Technology. 5 (3), 11 (2016).

40. Phillips, S. J. et al. Autologous transplantation of retinal pigment epithelium after mechanical debridement of 
Bruch's membrane. Current Eye Research. 26 (2), 81-88 (2003).

41. Sugita, S., Mandai, M., Kamao, H., Takahashi, M. Immunological aspects of RPE cell transplantation. Progress in Retinal \& Eye Research. (2021).

42. Xian, B., Huang, B. The immune response of stem cells in subretinal transplantation. Stem Cell Research \& Therapy. 6, 161 (2015).

43. Issa, F., Schiopu, A., Wood, K. J. Role of T cells in graft rejection and transplantation tolerance. Expert Review of Clinical Immunology. 6 (1), 155-169 (2010).

44. Yan, F. et al. Transforming growth factor- $\beta 2$ increases the capacity of retinal pigment epithelial cells to induce the generation of regulatory T cells. Molecular Medicine Reports. 13 (2), 1367-1372 (2016).

45. Singhal, S. et al. Chondroitin sulfate proteoglycans and microglia prevent migration and integration of grafted Müller stem cells into degenerating retina. Stem Cells. 26 (4), 1074-1082 (2008).

46. Singhal, S., Lawrence, J. M., Salt, T. E., Khaw, P. T., Limb, G. A. Triamcinolone attenuates macrophage/ microglia accumulation associated with NMDA-induced RGC death and facilitates survival of Müller stem cell grafts. Experimental Eye Research. 90 (2), 308-315 (2010).

47. Santa-Cecília, F. V. et al. Doxycycline suppresses microglial activation by inhibiting the p38 MAPK and NFkB signaling pathways. Neurotoxicity Research. 29 (4), 447-459 (2016).

48. Scholz, R. et al. Minocycline counter-regulates pro-inflammatory microglia responses in the retina and protects from degeneration. Journal of Neuroinflammation. 12, 209 (2015).

49. Sugita, S., Makabe, K., Iwasaki, Y., Fujii, S., Takahashi, M. Natural killer cell inhibition by HLA-E molecules on induced pluripotent stem cell-derived retinal pigment epithelial cells. Investigative Ophthalmology \& Visual Science. 59 (5), 1719-1731 (2018).

50. Sugita, S. et al. Detection of retinal pigment epitheliumspecific antibody in iPSC-derived retinal pigment epithelium transplantation models. Stem Cell Reports. 9 (5), 1501-1515 (2017). 